\title{
DESIGN AND IMPLEMENTATION OF IMAGE COMPRESSION USING SET PARTITIONING IN HIERARCHICAL TREES AND HYBRID WAVELET TRANSFORM
}

\author{
Jyoti V. Kadam ${ }^{1}$, Vijaykumar S. Kolkure ${ }^{2}$ \\ ${ }^{l}$ M.E. (Electronics, Appeared), Department Of Electronics Engineering,Bharatratna Indira Gandhi College of \\ Engineering,Affiliated to Solapur University, Solapur, Maharashtra, India. \\ ${ }^{2}$ Assistant Professor, Department Of Electronics Engineering, Bharatratna Indira Gandhi College of Engineering, \\ Affiliated to Solapur University, Solapur, Maharashtra, India.
}

\begin{abstract}
To store digital image and video in raw form require large amount of memory space. Image compression means reducing the size of image file without degrading quality of image. Depending on the reconstructed image to be exactly same as the original or some unknown loss may incurred image compression divided into two techniques lossy and lossless techniques. In this paper we present hybrid model which is the combination of several compression techniques. This paper present DWT, DCT and SPIHT implementation. Simulation has been carried out on different images like Lena, Barbra, Cameraman, Test drive. Result analysis is done through parameters like MSE, PSNR and Elapsed time. Values of this parameters will go better than the old algorithms because here we apply SPIHT lossless technique and also due to hybrid combination of DWT and DCT we will get good level of compression. The result analysis shows that the proposed hybrid algorithm performs much better in terms of PSNR with a higher compression ratio as compared to standalone DWT and DCT techniques.
\end{abstract}

Keywords- DWT, DCT, SPIHT, PSNR, MSE

\section{INTRODUCTION}

Image compression is storing images using lesser number of bits than its original size. Image compression leads to less storage space and less bandwidth for transmission. As compression ratio increases, quality of reconstructed image starts degrading. Many compression techniques are present but transform based techniques are popular for image compression. Discrete cosine transform (DCT) is widely used. It separates an image into different frequency components. Low frequency components are located at top left corner. Elimination of these high frequency elements gives transformed image with few low frequency components. If image if image is reconstructed from such lesser number of transformed low frequency elements, it gives compressed image without losing much data contents in original image. The DCT is fast. Discrete wavelet transform (DWT) has gained widespread acceptance in signal processing and image compression. The DWT is linear transformation that operates on a data vector whose length is an integer power of two, to transforming it into a numerically different vector of same length. It is tool that separates data into frequency components and then studies each component with resolution matched to its scale. Set Partitioning in Hierarchical Trees (SPIHT) is wavelet based image coder that has many good characteristics like it gives good image quality with a high value PSNR, fast coding and decoding process. SPIHT can be used as lossless compression. Wavelet transform coding is preferred over simple orthogonal transform in image compression due to its multi resolution property. It provides enhanced image quality even at higher compression ratios. In this paper we use hybrid transformation techniques which combine properties of two transforms i.e. DWT and DCT.

\section{PROPOSED SYSTEM}

Figure 1 shows the block diagram of proposed system. The input image used for this project is in different file formats (.bmp, .png, .jpg, .tif). The wavelet transform is applied on the original image i.e. image is subdivided into several frequency bands to analyze image data. DWT using different wavelets like Daubechies, Symlet and Biorthogonal is applied. Hybrid wavelet transform is obtained by combination of two orthogonal transform. Here, combination of DWT and DCT is used. Wavelet transform of size N2xN2 is generated from $\mathrm{NxN}$ transform matrix. After forward hybrid wavelet transform SPIHT encoder simply reduces the number of bits needed to store the transformed coefficients by reducing the precision of those values. SPIHT scans wavelet coefficients along quad-tree instead of sub band. SPIHT identifies significance only by the magnitude of wavelet coefficients and encodes the sign separately into a new bit stream. At the output of the encoder we get the compressed image. Compressed image is given as an input to the decoder. The decoder is a digital to analog converter which performs exactly opposite operation of the encoder. Here, inverse transform is applied on the decoded image which is an exact opposite operation of the forward hybrid wavelet transform. At the output of this stage we get the original reconstructed image. 


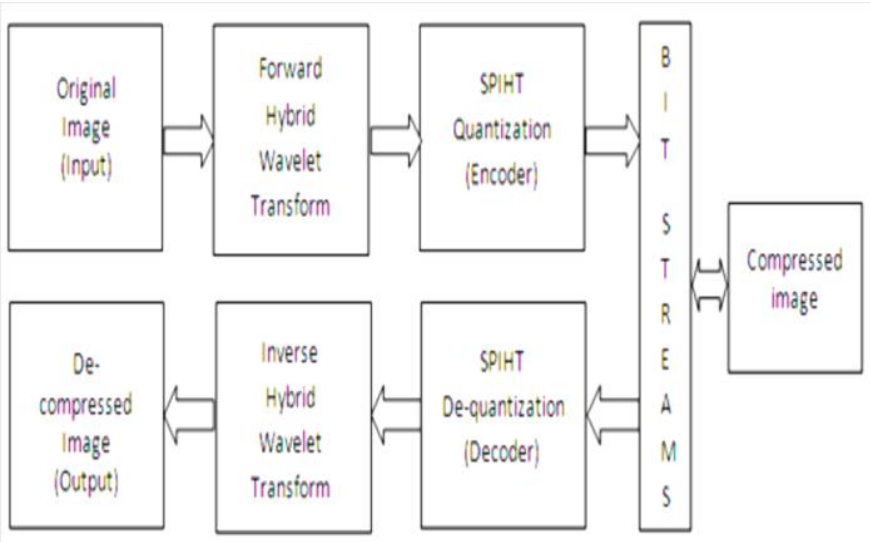

Fig 1: Block Diagram of Proposed System

\section{PERFORMANCE}

The compression performance is measured through parameters peak signal to noise ratio (PSNR), Mean Square Error, Elapsed time. The mathematical formulae for the same are

$$
\operatorname{MSE}=\operatorname{sum}\left(\operatorname{sum}\left((\operatorname{Im}-\mathrm{P}) .^{\wedge} 2\right)\right) /\left(\mathrm{m}^{*} \mathrm{n}\right)
$$

$\mathrm{PSNR}=10 * \log 10((255 * 255) / \mathrm{MSE})$

Where, Im is the original image, $\mathrm{P}$ is decoded image and $\mathrm{m}, \mathrm{n}$ are the dimensions of the image.

Elapsed time is the actual time between two events.

\section{RESULT ANALYSIS}

We have applied the hybrid (DWT, DCT) and SPIHT image compression algorithm on several images and result are shown in this section. Simulation is done by using MATLAB software. Table1. shows the result of values of parameters PSNR, MSE, Elapsed time for different images.

Table 1: Values of Different Parameters.

\begin{tabular}{|l|l|l|l|}
\hline IMAGE & PSNR & MSE & $\begin{array}{l}\text { ELAPSED } \\
\text { TIME(seconds) }\end{array}$ \\
\hline Lena & 33.82 & 26.94 & 11.80 \\
\hline Barbara & 33.25 & 30.71 & 13.68 \\
\hline Cameraman & 33.41 & 29.64 & 12.35 \\
\hline Test Drive & 32.77 & 34.33 & 14.21 \\
\hline
\end{tabular}

\section{CONCLUSION}

In this paper we design and implement a hybrid DWT, DCT and SPIHT algorithm for image compression. The performance analysis on different images shows that SPIHT and combination of DWT-DCT gives better result of image compression as compared to separate DWT and DCT techniques. Also these combination gives higher values of PSNR and lower values of MSE.

\section{REFERENCES}

[1]. Rachana Dhannawat, Tanuja Sarode, H. B. Kekre, "Kekre's Hybrid Wavelet Transform Technique with DCT, Walsh, Hartley and Kekre's Transform for Image Fusion", International Journal of Computer Engineering and Technology(IJCET), Vol. 4, Issue 1, Feb 2013, pp. 195-202.

[2]. JPEG2000 Image Coding System, document ISO/IEC 15444-1, 2000.

[3] .J. M. Shapiro, D. S. R. Center, and N. J. Princeton, "Embedded image coding using zerotrees of wavelet coefficients", IEEE Signal Process. vol. 41, no. 12, pp. 34453462, Dec. 1993.

[4]. D. Taubman, "High performance scalable image compression with EBCOT", IEEE Trans. Image Process., vol. 9, no. 7, pp. 11581170, Jul. 2000.

[5]. A. Said and W. Pearlman, "A new, fast, and efficient image codec based on set partitioning in hierarchical trees", IEEE Trans. Circuits Syst. Video Technol., vol. 6, no. 3, pp. 243250, Jun. 1996.

[6]. G. Pastuszak, "A novel architecture of arithmetic coder in JPEG2000 based on parallel symbol encoding", in Proc. Int. Conf. Parallel Comput. Electr. Eng., 2004, pp. 303308.

[7]. Dr. H. B. Kekre, Tanuja Sarode, Prachi Natu, "Performance Comparison of Hybrid Wavelet Transform Formed by Combination of Different Base Transforms with DCT on Image Compression", International Journal of Innovative Research in Science, Engineering and Technology, Volume 3, Issue 1, January 2014.

[8]. Bhonde Nilesh, Shinde Sachin, Nagmode Pradip, D. B. Rane, "Image compression Using Discrete Wavelet Transform", International Journal of Computer Technology and Electronics Engineering (IJCTEE) Volume 3, Special Issue, March-April 2013.

[9]. Moh dAli Moustafa Alsayyh, Prof. Dr. Dzulkii Mohamad, "Image Compression Using Hybrid Technique", Information and Knowledge Management ISSN 2224- 5758 (Paper) ISSN 2224-896X (Online)Vol 2, No.7, 2012.

[10]. J. Maly, P. Rajmic, "DWT-SPIHT Image Codec Implementation", IEEE Transactions, 2012, pp. 340-344. 44

[11]. Mr. M.B. Bhammar, Prof. K. A. Mehta, "Performance Improvement Of SPIHT Algorithm Using Hybrid Image

\section{BIOGRAPHIES}

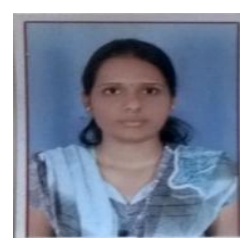

Ms. Jyoti Vishnu Kadam ${ }^{1}$ currently pursuing M.E. (Electronics) From Bharatratna Indira Gandhi College of Engineering, Solapur, Maharashtra, India. Her area of interest is image processing.

Vijaykumar S. Kolkure ${ }^{2}$ has completed M.E. (Electronics) from W.C. Sangli, Maharashtra, India. He has 10 years of teaching experience. Currently he is working as Assistant Professor at Bharatratna Indira Gandhi College of Engineering, Solapur, Maharashtra, India. His area of interest is Image Processing, Video Processing. 
Section below shows the simulation result on Lena, Barbara, Cameraman and Test drive. For this purpose we have taken image size of $256 * 256$.


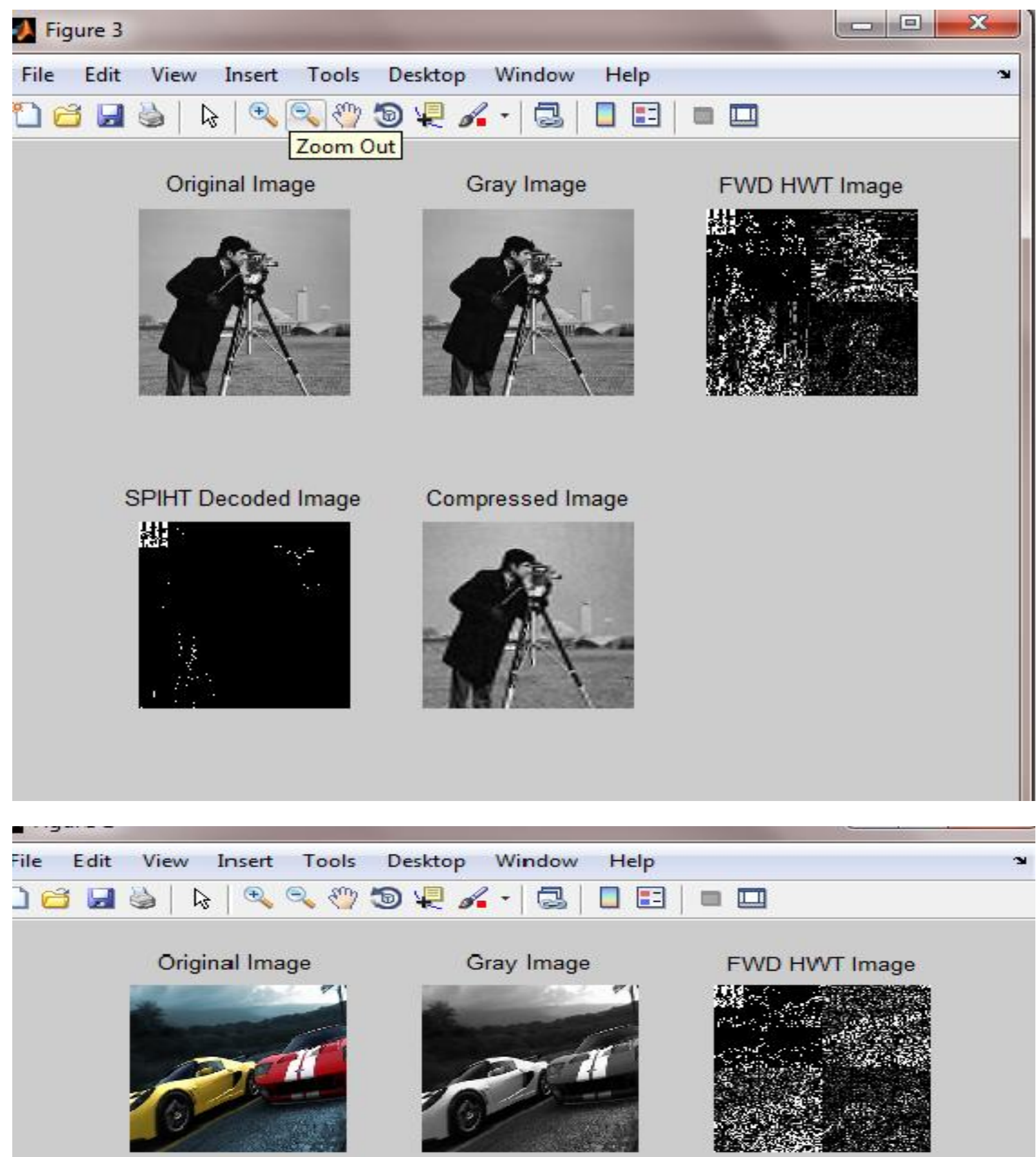

FWD HWT Image
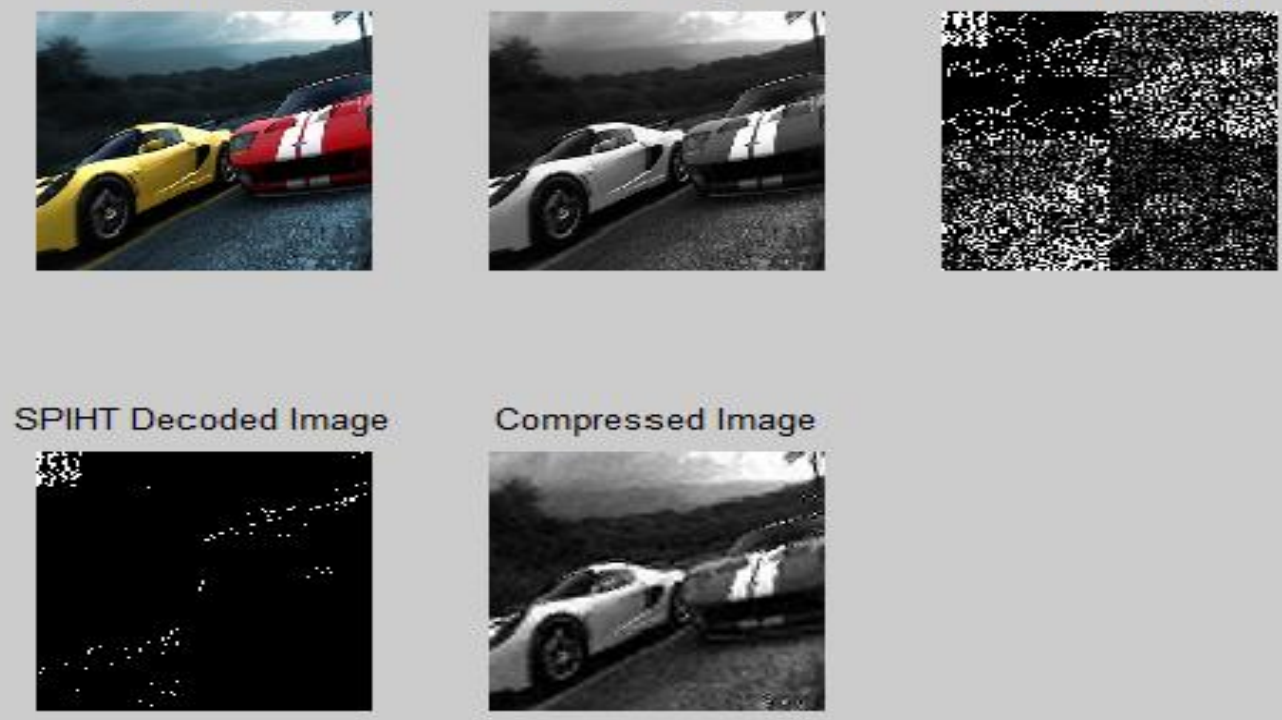